Bericht: Specielle analyt. Methoden. 1. Auf Lebensmittel etc. bezügl. 721

IV. Specielle analytische Methoden.

1. Auf Lebensmittel, Gesandheitspflege, Handel, Industrie und Landwirthschaft bezügliche.

Von

\title{
I. Grünhut.
}

Verfälschung gemahlener Gewürze mit Rohrzucker. Gemahlene Gewürze werden häufig mit den extrahirten, ihres ätherischen Oeles beraubten Gewürzen gleicher Art verfälscht, die in den Fabriken ätherischer Oele als Rückstände gewonnen werden. Um den herben, bitteren und zusammenziehenden Geschmack solcher Mischungen zu verdecken, wird ihnen dann noch Rohrzucker zugesetzt. Ed. Spaeth ${ }^{1}$ ) fand in gemahlenem Zimmt bis zu $10 \%$, in Macis bis zu $20 \%$ desselben auf. Der Nachweis und die quantitative Zuckerbestimmung geschieht polarimetrisch. $20 \mathrm{~g}$ Zimmtpulver oder Macispulver werden mit $100 c c$ Wasser versetzt und einige Minuten damit geschüttelt. Nach 10 Minuten (nicht länger) wird dieses Durchschütteln wiederholt; dann filtrirt man durch ein Faltenfilter. Einen Theil des Filtrat versetzt man mit $1 / 10$ Volumen Bleiessig und Thonerdebrei und verwendet die geklärte Flüssigkeit zur Polarisation. Reine Macis zeigt bei dieser Behandlung gar keine Drehung, eben so wenig chinesischer Zimmt, Zimmtcassie und Holzcassie. Ceylonzimmt enthält dagegen 0,50 bis $1,56 \%$ Invertzucker and weist in Folge dessen eine Linksdrehung von 0,1 bis 0,2 Kreisgraden im $220 \mathrm{~mm}$-Rohr auf.

Beim Schütteln des Zimmts oder der Macis mit Chloroform fällt übrigens der beigemischte Zucker rasch zu Boden und kann in Substanz isolirt werden. Man bedient sich hierbei am besten des vom Verfasser beschriebenen Sedimentirglases. ${ }^{2}$ )

Eine neue Methode der Fettbestimmung in Molkereiproducten gründet Lindet ${ }^{3}$ ) auf die von $\mathrm{Tsvett}^{4}$ ) beobachtete Löslichkeit des Caseïns in concentrirter wässriger Resorcinlösung. In eine graduirte Röhre, welche unterhalb des Nullpunktes ihrer Theilung bauchig erweitert ist, und von welcher das Original eine nähere Beschreibung

1) Forschungsberichte über Lebensmittel etc. 3, 291.

2) Vergl. diese Zeitschrift 37, 320.

3) Bull. de la soc. chim. de Paris (3. sér.) 23, 409.

4) Comptes rendus 129, 551 .

Fresenius, Zeitsohrift f. analyt. Chemie. XXXIX. Jahrgang. 11. Heft. 48 
enthält, bringt man $5 g$ Resorcin, $5 c c$ Milch und 2 Tropfen Natronlauge von $36^{\circ}$ Bé. Dann führt man den ganzen Apparat in ein siedendes Wasserbad ein und liest das Volumen des anf der Oberfläche abgeschiedenen Fettes bei Wasserbadtemperatur mit Hülfe der Theilungder Röbre ab. $1 g$ Milchfett nimmt ein Volumen ein, das, bei $+15^{0} \mathrm{C}$. gemessen, 1,154 cc betragen würdo. Rahm muss behufs Fettbestimmung nach dieser Methode zuvor mit Wasser verdünnt werden and wird dann wie Milch behandelt. Zur Fettbestimmung im Käse behandelt man $1 \mathrm{~g}$ desselben mit $15 \mathrm{cc}$ einer warmen 50 procentigen, wässrigen Resorcinlösung.

Butteruntersuchung. Butterschmalz wird in Oberfranken hier und da abgekocht, da man glaubt hierdurch einem Verderben besser vorbeagen zu können. Mit Rücksicht hierauf untersuchte Ed. Spa eth ${ }^{1}$ ) die Veränderungen, welche Butterschmalz and andere Fette beim Erhitzen erleiden. Die Ergebnisse lassen sich in folgende Sätze zusammenfassen:

1. Die Verseifungszahl und Refractometerzahl stärker erhitzter oder gekochter Fette ist höher als diejenige der normalen Fette. Die Reichert-Meissl'sche Zahl wird wenig oder gar nicht beeinflusst. Die Jodzahl wird erniedrigt, and zwar bei Fetten von nicht zu hoher Jodzahl (Butterschmalz, Schweinefett, Margarine) nur um ein geringes, bei Fetten von hoher Jodzahl (Oelen etc.) dagegen um einen erheblichen Betrag.

2. Erhitzte Fette zeigen ein ähnliches Verhalten wie Fette, die ranzig werden. In stark ranzigen Fetten wird nach dem Erhitzen derselben die Verseifungszahl wieder niedriger als in den nicht erhitzten, bleibt aber höher als in den normalen Fetten. Jodzahl und Brechungsindex werden in der bekannten Weise beeinflusst. Ein besonderer Einfluss auf die Reichert-Meissl'sche Zahl konnte bei ranzig gewordenem Butterschmalz nicht bemerkt werden.

3. In Beziehuug auf die festgestellte Thatsache, dass sowohI bei ranzigem, wie auch stark erhitztem Butterschmalz erhöhte Verseifungszahlen vorkommen, erscheint es geboten bei der Untersuchung von Butterschmalz der Meissl'schen Probe den Vorzug zu geben.

Im Gegensatz hierzu legten R. Hefelmann und P. Mann ${ }^{2}$ ) bei der Beurtheilung der Butter weit grösseren Werth auf die Köttstorfer-

1) Zeitschrift f. Untersuchung der Nahrungs- u. Genussmittel 1, 377.

2) Pharm. Centralhalle $\mathbf{3 6}, 231$. 